\title{
MICRO-FIELDS OF SHORT FIBRE IN MATRIX AND MACRO-DYNAMIC RESPONSE OF FIBRE COMPOSITES
}

\author{
Z. Murčinková*
}

\begin{abstract}
The paper analyses the short-fibre composites, their mechanical and thermal micro-fields determined by numerical simulation by both p-FEM and specific numerical mesh-reducing method focusing on shape, size, volume fraction, material properties ratio. Moreover, the paper provides the experimental results and comparison of macro-dynamic response measuring (damping time, natural frequencies) for standard materials and the short-fibre composites and analyses the FFT (Fast Fourier Transformation) spectrums of materials.
\end{abstract}

Keywords: short fibre composite, mechanical and thermal micro-fields, damping

\section{Introduction}

The real not-idealized materials involve different types of in-homogeneities. The in-homogeneities, i.e. holes, cavities, cracks, inclusions, created during technological production process act as the stress concentrators and cause various local effects of large field gradients. Naturally, we recognize the stress concentrators as undesirable factors (Frydrýšek and Lenert, 2004). Despite that fact, the short-fibre composites involve "the in-homogeneities", i.e. short fibres, but in role of the reinforcing elements causing the improvement of the material properties (Žmindák and Dudinský, 2012). The useful combination of matrix and reinforcing elements their shape, size, interphase, volume fraction, material properties ratio, arrangement etc. allow to form the new material - composite material of improved required properties.

\section{Static response of short-fibre and matrix}

The starting point for identifying and understanding the micro-field distribution of the short-fibre composite materials is model of single fibre in matrix. For numerical simulation of static response, the pFEM was used. The results were confirmed by boundary meshless-type method named method of continuous source functions (more in Kompiš et al, 2015) developed for short fibres. In the longitudinal direction, the fibre length dimension is several orders larger. The aspect ratio, AR, (fibre length:diameter) can be e.g. 200 000. So, we can call short fibres as relatively short regarding large AR.

The numerical model is the square unit cell (UC) involving short fibre and matrix. As the advantage of UC symmetry of geometry and boundary conditions is used, the one eight of UC is modelled and computed. The constant prescribed displacement in the direction of fibre is defined. The assumptions of numerical model are: The fibre is straight and cylindrical; the fibres are regularly distributed without overlapped configuration; the end of each fibre is modelled as half-sphere to avoid singularity; the fibre and matrix create the continuous material without gaps; the material of both matrix and fibre is considered to be isotropic linear elastic; any interface layer is not assumed between fibre and matrix; the ideal cohesion between matrix and fibre is assumed.

assoc. prof. Zuzana Murčinková, PhD.: Faculty of Manufacturing Technologies with seat in Prešov, Technical university of Košice, Bayerova 1; 08001, Prešov; SK, zuzana.murcinkova@tuke.sk 

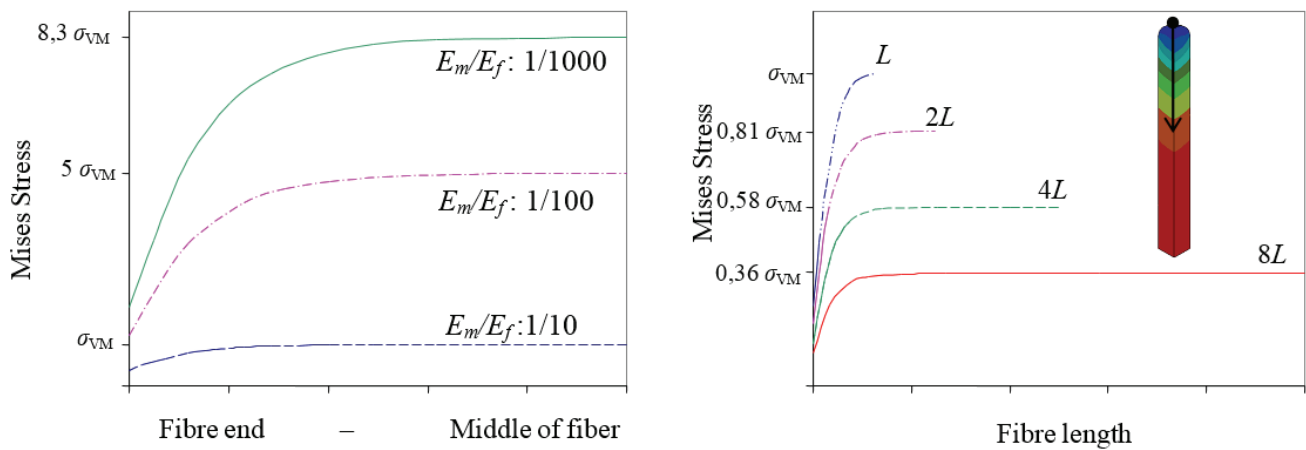

Fig. 1: Mises Stress of fibre L50/R1 for various Em/Ef (left) and Mises Stress due to fibre length, Em/Ef: 1/100 (right)

Fig. 1, left, presents the sensitivity of short fibre composite to matrix and fibre material properties, namely $E_{m}$ and $E_{f}$ is Young modulus of matrix and fibre, respectively. Naturally, the larger difference in fibre and matrix material properties, the larger stress in fibre. But if the ratio $E_{m} / E_{f}$ (compare to $1: 10$ and 1:1000) is larger 100-times, the Mises stress is larger only 8.3-times keeping the same dimensions and boundary conditions. The stiffer fibre in matrix improves stiffness but it makes undesirable the larger shear stress on fibre-matrix interface.

Fig. 1, right, shows the relationship between fibre length and Mises Stress. The material properties and boundary condition are the same for each numerical model. The graph involves distribution of Mises Stress in one eight of short fibre situated in matrix. The middle part of fibre carries the largest stress. If the fibre length is 8-times larger (compare L25 and L200), the Mises stress is 2.7-times lower. The relatively long fibre in matrix is more suitable than short (of small AR).

Fig. 2 provides the influence of another parameter, i.e. volume fraction. The more fibres in volume, the lower stress in each fibre. If the volume fraction is 7-times larger, the Mises stress is only 3.75-times lower.

Both the fibre ends and fibre-matrix interface are critical but important locations of short fibre composite regarding its strength and material properties. The largest shear stress is in the fibre ends on fibre surface (Fig. 3). The large micro-fields gradients in matrix caused by adjacent fibre are visible in Fig. 4, left. The maximum Mises stress in matrix is placed near fibre end. Thus the fibre ends and their closest neighbourhood involving fibre-matrix interface are crucial regarding static, thermal as well as dynamic properties. Maximum shear stress between the fibre and the matrix can result in de-bonding of the fibres

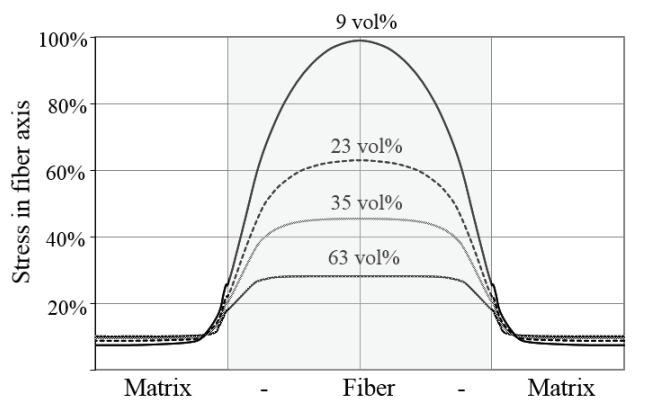

Fig. 2: Mises stress in fibre axis due to change of fibre volume fraction; fibre AR is 20

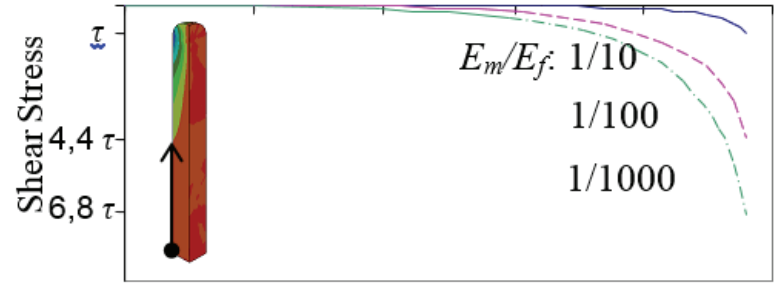

Middle of fibre

Fibre end

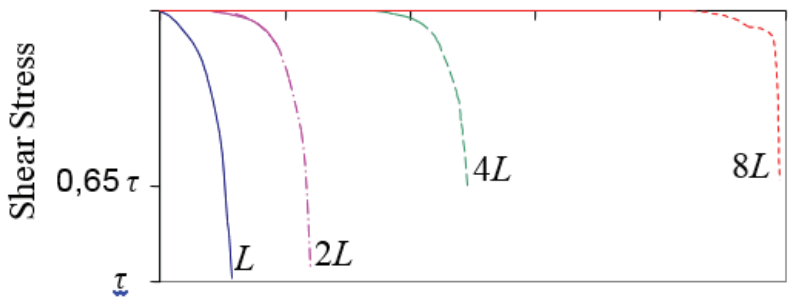

Middle of fibre
Fibre end

Fig. 3: Shear Stress on fibre/matrix interface (fibre AR is 25) for various Em/Ef (left) and Shear Stress regarding fibre length Em/Ef: 1/100 (right) 

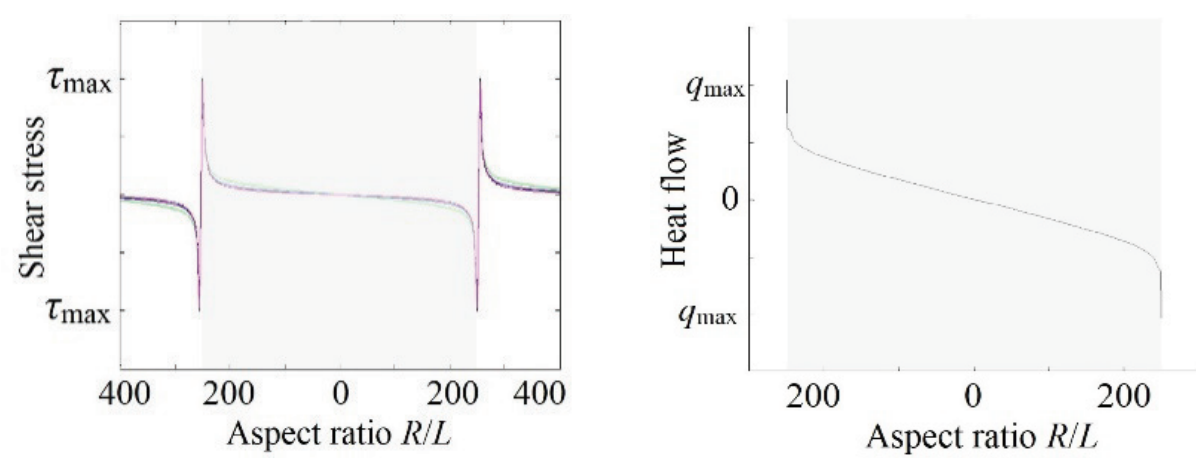

Fig. 4: Shear stress (left) and heat flow in direction perpendicular to fiber axis (right), fiber AR is 500

or to de-cohesion and re-cohesion at the ends. Also, the mentioned effects can appear anywhere on the fibre-matrix interface along fibre as the increasing of stress can be caused by the fibre end of neighbouring fibre.

The elasticity and strength limit of short-fibre composite material depend on the connectivity between fibre and matrix, not only on suitable combination of matrix/fibre materials. However, the aim is not to make the stiff connection at the fibre ends to avoid the very high gradients of shear stress there. Nevertheless, the effect of de-cohesion and re-cohesion at the ends of fibres are appropriate regarding it contributes to very fine material damping.

\section{Spherical elements in particulate composite}

In case we make the same analysis for influence of spherical elements in particulate composite on stress distribution, some of relationships would have the opposite slope, i.e. the larger volume fraction of particulate "reinforcing" element, the larger stress. Therefore, the spherical particulate composites are designed not to improve strength but other unusual combination of properties as low weight and better damping properties.

\section{Analogy - mechanical and thermal micro-fields}

Fig. 4 presents analogy between the distribution of shear stress and heat flow perpendicular to fiber axis. It is an example of analogy between mechanical and thermal micro-fields: normal stress in fibre axis and heat flow in fibre axis; displacement in fibre direction and temperature, etc. Both fields in Fig. 4 are obtained by specific numerical method intended for short-fibre composites named Method of continuous source functions (more in Kompiš and Murčinková, 2014, Kompiš et al., 2015).

\section{Macro-dynamic response}

Dynamic response of material is important in many applications, mainly in the most demanding problems such as aerospace but also in special applications in mechanical or civil engineering industry. Dynamic response of material is in form of mechanical waves (vibrations) that propagate in continuum. Vibration is the oscillation of elastic body or environment where individual particles make mechanical oscillation.

If the object is impacted with a massive object such as a hammer (the bump test), the resulting spectrum contains peaks that correspond to the natural frequencies or 'resonances' of object. The measuring was made in radial direction (bending), twice per each sample (two perpendicular directions). The tested samples were pipes with dimensions: outer diameter $D=12 \mathrm{~mm}$, inner diameter $d=8 \mathrm{~mm}$, length $L=110 \mathrm{~mm}$. The samples were made of carbon steel, aluminum alloy (duralalumin), glass $\left(\mathrm{SiO}_{2}\right)$, corundum (oxide ceramics $\mathrm{Al}_{2} \mathrm{O}_{3}$ ) and $\mathrm{C} / \mathrm{SiC}$ composite (ceramics matrix with carbon short fibers). The measured values are in Fig. 5. The samples are different for their material and microstructure. Material properties of $\mathrm{C} / \mathrm{SiC} \perp$ samples are directional. In Fig. 5 the letter $\mathrm{N}$ means narrow (Fig. 6 b,d) and $\mathrm{W}$ wide (Fig. 6 a,c) frequency zone.

The steel and aluminum alloy represent conventional materials. Glass $\left(\mathrm{SiO}_{2}\right)$ is representative of amorphous, isotropic, solid and brittle material in metastabile state. The samples made of ceramics composite $\mathrm{C} / \mathrm{SiC}$. $\mathrm{SiC}$ (carborundum) belongs to technical non-oxide ceramics branch. $\mathrm{SiC}$ are infiltrated by short carbon fiber that improving the mechanical and thermal properties of SiC. Short-fibre composite $\mathrm{C} / \mathrm{SiC}$ comprises short carbon fibers with length of 3 to $6 \mathrm{~mm}$ of $12 \mathrm{k}$ thickness ( $1 \mathrm{k}=1000$ filaments). The $\mathrm{C} / \mathrm{SiC}$ and $\mathrm{C} / \mathrm{SiC} \perp$ samples have different orientation of fibers in volume. The $\mathrm{C} / \mathrm{SiC}$ sample has 

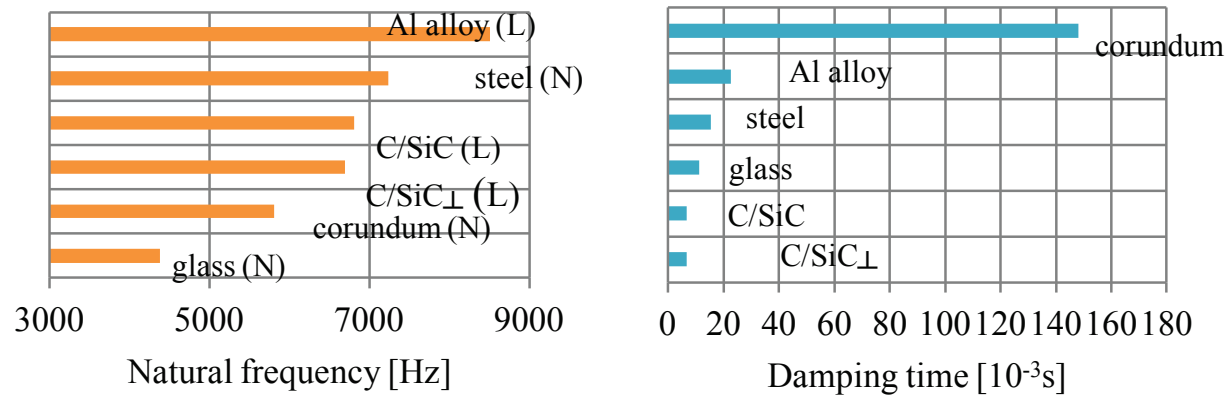

Fig. 5: Natural frequency and damping time

a)

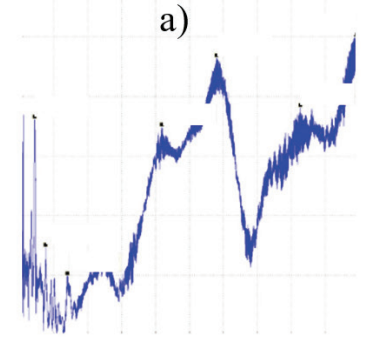

b)

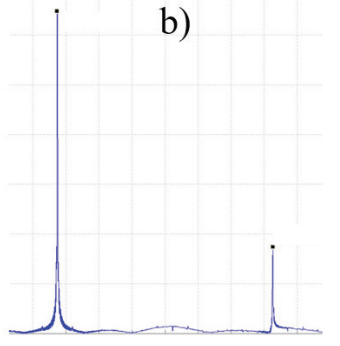

c)

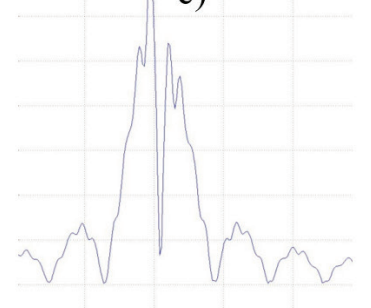

d)

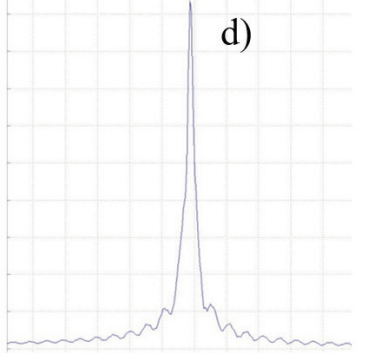

Fig. 6: Character of FFT spectrum of layered long fibres (laminate), (a), short fibre composite (b), steel (c) and aluminium alloy (d)

randomly distributed fibers so the material can be considered to be isotropic. The $\mathrm{C} / \mathrm{SiC} \perp$ sample has short fibers with preferred orientation perpendicular to pipe axis. The material properties $\mathrm{C} / \mathrm{SiC} \perp$ sample can be recognized as orthotropic.

Fig. 6 shows typical shape of narrow and wide frequency zone of dynamic response. The dominant natural frequency is not evident for aluminium alloy, $\mathrm{C} / \mathrm{SiC}$ and $\mathrm{C} / \mathrm{SiC} \perp$ samples. Such property is very suitable for preventing the resonance comparing with steel, $\mathrm{SiO}_{2}$ and $\mathrm{Al}_{2} \mathrm{O}_{3}$ samples that have narrow frequency zone with one dominant natural frequency. $\mathrm{Al}_{2} \mathrm{O}_{3}$ sample has several times longer damping time comparing with others. If the non-damped $\mathrm{Al}_{2} \mathrm{O}_{3}$ sample (or component) would be excited by dynamic force, the vibrations would be larger. One can see that damping time is very short in case of composite material samples. Such materials are very appropriate for designing the components intended for dynamic load with variable exciting frequencies.

\section{Conclusions}

The short-fibre composites are characterized by specific relations between their parameters as shape, size, volume fraction, material properties ratio, etc. The paper analysed the mentioned parameters through the micro-mechanical numerical analysis with aim to understand the behaviour of short fibres as reinforcing elements (not undesirable inclusions). The dynamic response of layered laminate and steel is characterized by one sharp peak comparing to several peaks of short fibre composite and aluminium alloy that form the wide frequency range without significant frequencies.

\section{Acknowledgement}

Authors thank for supporting this research by grant VEGA 1/0910/17 of Agency of Ministry of education of Slovak Republic.

\section{References}

Frydrýšek, K. and Lenert, J. (2004). Mechanics of Materials: (strength of Materials): Compendium. VŠB-Technical University of Ostrava.

Kompiš, V. and Murčinková, Z. (2014). Thermal properties of short fibre composites modeled by meshless method. Advances in Materials Science and Engineering, 2014.

Kompiš V, Murčinková Z and Žmindák M (2015) Toughening mechanisms for the fibre of middle-large-aspectratio-reinforced composites. In: Toughening mechanisms in composite materials, Qin, Q.H. and Ye J. (eds.), Cambridge: Elsevier; Woodhead Publishing, chapter 5, pp 137-159.

Žmindák, M. and Dudinský, M. (2012). Computational modelling of composite materials reinforced by glass fibers. Procedia engineering, 48, 701-710. 